\title{
GERMINAÇÃO DE SEMENTES DE Dypsis decaryi (Jum.) Beentje \& J. Dransf. (Arecaceae)
}

\author{
Germination of Dypsis decaryi (Jum.) Beentje \& J. Dransf. (Arecaceae) seeds
}

\author{
Petterson Baptista da Luz ${ }^{1}$, Ricardo Soares Pimenta ${ }^{2}$, Patricia Unger César Pizetta ${ }^{3}$, \\ Amanda de Castro ${ }^{4}$, Kathia Fernandes Lopes Pivetta ${ }^{5}$
}

\begin{abstract}
RESUMO
Dypsis decaryi (Jum.) Beentje \& J. Dransf. é uma importante palmeira comercializada para fins ornamentais. Embora seja propagada sexuadamente, não há informações na literatura sobre os fatores que afetam a germinação das sementes dessa espécie. Objetivou-se, neste trabalho estudar o efeito da temperatura e do substrato na germinação de sementes de Dypsis decaryi. O delineamento experimental foi o inteiramente casualizado em esquema fatorial 6 × 2 (seis temperaturas: $25^{\circ} \mathrm{C}, 30^{\circ} \mathrm{C}, 35^{\circ} \mathrm{C}, 20-30^{\circ} \mathrm{C}$ e $25-35^{\circ} \mathrm{C}$ e ambiente combinadas com dois substratos: vermiculita e areia), com 5 repetições de 20 sementes. Anotou-se, diariamente, o número de sementes germinadas adotando como critério de germinação a protrusão do botão germinativo. Após 35 dias houve estabilização dos dados e foi calculado o Índice de Velocidade de Germinação (IVG) e a porcentagem de germinação (dados transformados em arc sen $\sqrt{x / 100}$ ) cujas médias foram comparadas pelo teste de Scott-Knott, a 5\% de probabilidade. Observou-se que maiores porcentagens de germinação de sementes de Dypsis decaryi foram obtidas quando colocadas para germinar em areia nas temperaturas ambiente de laboratório $(93 \%), 25-35^{\circ} \mathrm{C}(92 \%)$ e $25^{\circ} \mathrm{C}(86 \%)$, e em vermiculita, nas temperaturas de ambiente $(91 \%), 20-30^{\circ} \mathrm{C}(87 \%)$, $30^{\circ} \mathrm{C}(83 \%), 25-35^{\circ} \mathrm{C}(80 \%)$ e $25^{\circ} \mathrm{C}(76 \%)$. As sementes germinaram mais rápido em condições de ambiente de laboratório independentemente do substrato. Na temperatura de $35^{\circ} \mathrm{C}$ a porcentagem de germinação foi bastante reduzida tanto em areia $(21 \%)$ como em vermiculita (38\%); as sementes germinaram mais lentamente e a germinação foi desuniforme.
\end{abstract}

Termos para indexação: Palmeira, propagação sexuada, teste de germinação, Dypsis decaryi.

\begin{abstract}
Dypsis decaryi (Jum.) Beentje \& J. Dransf. is an important palm with ornamental commercial value. Although it reproduces sexually, there are no data available as far as what are the factors that affect this species seed germination. The purpose of this work is to study the potential effects of temperature and substrate in Dypsis decaryi seed germination. The research framework is based on randomization, using a $6 \times 2$ factorial schema (six different temperatures, $25^{\circ} \mathrm{C}, 30^{\circ} \mathrm{C}, 35^{\circ} \mathrm{C}, 20-30^{\circ} \mathrm{C}, 25-35^{\circ} \mathrm{C}$ and room temperature, combined with two types of substratum, sand and vermiculite) with 5 repetitions of 20 seeds. The researcher made daily records on the number of germinated seeds, and the criteria used was protusion of the germination plug. After 35 days data become stable. The Index of Germination Speed (IVG) and germination percentage were then calculated (data were converted to sen). The averages for these two indexes were compared using the Scott-Knott method with a probability of $5 \%$. The data show that the higher percentages of germination were obtained with a sand substratum at temperatures of $25^{\circ} \mathrm{C}(86 \%), 25-35^{\circ} \mathrm{C}(92 \%)$ and lab room temperature $(93 \%)$, and with a vermiculite substratum, at temperatures of $25^{\circ} \mathrm{C}(76 \%), 30^{\circ} \mathrm{C}(83 \%), 20-30^{\circ} \mathrm{C}(87 \%), 25-35^{\circ} \mathrm{C}(80 \%)$ and room temperature $(91 \%)$. The seeds germinated sooner in the lab environment, for both substrates. The percentage of germination at $35^{\circ} \mathrm{C}$ was markedly lower, both on sand $(21 \%)$ and vermiculite $(38 \%)$. In this case, the seeds took longer to germinate and germination was not uniform.
\end{abstract}

Index terms: Palm, sexual reproduction, germination study, Dypsis decaryi.

(Recebido em 27 de outubro de 2006 e aprovado em 30 de junho de 2008)

INTRODUÇÃO

Nativa de Madagascar, a espécie Dypsis decaryi (Jum.) Beentje \& J. Dransf., conhecida como palmeiratriângulo, é utilizada em parques e jardins, isolada, em grupo ou em fileiras. A característica singular de distribuição trística das folhas e cor acinzentada são seus principais atributos ornamentais (LORENZI et al., 2004).

As palmeiras, com poucas exceções são propagadas por meio de sementes que apresentam germinação lenta e desigual e é influenciada por vários fatores, como estádio de maturação, presença ou não de pericarpo, tempo entre

\footnotetext{
Engenheiro Agrônomo, Doutor em Produção e Tecnologia de Sementes, Professor - Departamento de Agronomia - Universidade do Estado do Mato Grosso/UNEMAT - Campus Universitário de Cáceres - Avenida São João, s/n - Cavalhada - 78200-000 - Cáceres, MT - petterbaptista@yahoo.com.br 'Biológo, Doutorando em Produção e Tecnologia de Sementes - Departamento de Produção Vegetal - Universidade Estadual Paulista/UNESP - Via de Acesso Professor Paulo Donato Castellane, s/n - 14884-900 - Jaboticabal, SP - pimenta@fcav.unesp.br

${ }^{3}$ Engenheira Agrônoma, Doutora em Produção Vegetal - Departamento de Produção Vegetal - Universidade Estadual Paulista/UNESP - Via de Acesso Professor Paulo Donato Castellane, s/n - 14884-900 - Jaboticabal, SP - patpizetta@yahoo.com.br

${ }^{4}$ Engenheira Agrônoma - Departamento de Produção Vegetal - Universidade Estadual Paulista/UNESP - Via de Acesso Professor Paulo Donato Castellane, s/n - 14884-900 - Jaboticabal, SP - amandinha-castroo@hotmail.com

${ }^{5}$ Engenheira Agrônoma, Doutora em Produção Vegetal, Professora - Departamento de Produção Vegetal - Universidade Estadual Paulista/UNESP - Via de Acesso Professor Paulo Donato Castellane, s/n - 14884-900 - Jaboticabal, SP - kathia@fcav.unesp.br
} 
colheita e semeadura, dormência física, temperatura do ambiente e substrato (MEEROW, 1991).

De acordo com Bewley \& Black (1985), a temperatura influencia tanto a porcentagem final de germinação como a velocidade de germinação. As sementes são capazes de germinar sob uma determinada amplitude de temperatura, definida para cada espécie, existindo uma temperatura máxima e uma mínima, acima e abaixo das quais a germinação não ocorre. Para as espécies da Família Arecaceae, há variações de temperatura desde 24 até $35^{\circ} \mathrm{C}$ (BROSCHAT, 1994; LORENZI et al., 2004; MEEROW, 1991).

O substrato para ser utilizado em testes de germinação deve preencher certos requisitos como ser atóxico à semente, ser isento de microorganismos e manter uma proporção adequada entre disponibilidade de água e aeração (COPELAND \& MCDONALD, 1995). A escolha do substrato, conforme consta em Brasil (1992), tem que ser feita em função da espécie e considerando algumas das características, tais como tamanho das sementes, necessidade de água e luz e, ainda facilidade da contagem e avaliação das plântulas.

Com relação ao substrato utilizado em testes de germinação Figliolia et al. (1993) comenta, ainda, que é importante mantê-lo uniformemente úmido, a fim de suprir as sementes com a quantidade de água necessária para sua germinação e desenvolvimento. O excesso de umidade provoca um decréscimo da germinação, pois impede a penetração de oxigênio e reduz todo o processo metabólico resultante, além de aumentar a incidência de fungos, levando à redução da viabilidade.

Yocum (1964) referiu-se à vermiculita como um substrato adequado para a germinação de palmeiras, por ser livre de pragas e doenças, ter boa drenagem e capacidade de retenção de umidade. Segundo observação do autor, a maneira conveniente de inserção das sementes no meio dependerá da forma dessas sementes. Aquelas com formatos elipsóides ou ovóides devem ser dispostas horizontalmente, enquanto as arredondadas não dispensam maiores cuidados. É importante o conhecimento da região da micrópila, segundo o autor, ao fazer a semeadura, as sementes devem ficar com a metade exposta acima da superfície do meio de germinação.

Pelo grande interesse ornamental e comercial e a escassez de relatos na literatura sobre a germinação das sementes de Dypsis decaryi, objetivou-se, neste trabalho, estudar o efeito da temperatura e do substrato na germinação de sementes dessa espécie.

\section{MATERIAL E MÉTODOS}

Frutos de Dypsis decaryi foram coletados de exemplares existentes na Coplana, Jaboticabal, em janeiro de 2006. O experimento foi instalado logo após a colheita e conduzido no Laboratório de Análise de Sementes do Departamento de Produção Vegetal.

$\mathrm{O}$ delineamento experimental foi o inteiramente casualizado em esquema fatorial 6 × 2 (6 temperaturas: ambiente; constantes de $25^{\circ} \mathrm{C}, 30^{\circ} \mathrm{C}$ e $35^{\circ} \mathrm{C}$ e alternadas de $20-30^{\circ} \mathrm{C}$ e $25-35^{\circ} \mathrm{C}$ com fotoperíodo de 8 horas de luz e 16 horas de escuro, combinadas com dois substratos: vermiculita e areia), com 5 repetições de 20 sementes.

$\mathrm{Na}$ condição de ambiente, os diásporos foram colocados sobre bancadas do laboratório, cujas temperaturas máximas e mínimas foram monitoradas diariamente, sendo a temperatura máxima média de $27,5^{\circ} \mathrm{C}$ e mínima média de $24,5^{\circ} \mathrm{C}$ e fotoperíodo de 8 horas de luz.

Após a colheita, o pericarpo e o mesocarpo dos frutos foram removidos por meio de atrito manual contra uma peneira e os diásporos, constituídos de endocarpo e semente, enxaguados em água corrente e secos à sombra, durante um dia.

Os diásporos foram colocados em caixas plásticas transparentes com tampa (tipo gerbox) nas dimensões de $11 \times 11 \times 3 \mathrm{~cm}$, contendo vermiculita média ou areia de rio lavada e esterilizada, conforme o tratamento. A reposição de água foi feita semanalmente mantendo capacidade de campo de $100 \%$, para ambos os substratos. As regas foram realizadas utilizando água destilada com $0,2 \%$ de nistatina para se evitar a contaminação por fungos.

A temperatura ambiente foi monitorada diariamente por meio de termômetros de máxima e mínima, sendo $29^{\circ} \mathrm{C}$ a temperatura máxima alcançada e $24^{\circ} \mathrm{C}$ a mínima.

Anotou-se diariamente o número de sementes germinadas adotando como critério de germinação a protrusão do botão germinativo. O teste foi conduzido até a estabilização da germinação, que ocorreu aos 40 dias.

Determinou-se a porcentagem de germinação e o Índice de Velocidade de Germinação (IVG). A porcentagem de germinação foi calculada pela fórmula proposta nas Regras para Análise de Sementes (BRASIL, 1992) e o IVG de acordo com a fórmula proposta por Maguire (1962).

Os dados foram submetidos à análise de variância com auxílio do programa SISVAR ${ }^{\circledR}$ (FERREIRA, 2000), e as médias comparadas pelo teste de Scott-Knott, a 5\% de probabilidade, sendo que os dados de porcentagem de germinação foram transformados em arc sen $\sqrt{x / 100}$. 


\section{RESULTADOS E DISCUSSÃO}

Analisando os resultados do Índice de Velocidade de Germinação - IVG, observa-se que a interação entre temperatura e substrato não foi significativa. Houve efeito altamente significativo da temperatura sendo que as sementes germinaram mais rápido em ambiente de laboratório e mais lentamente na temperatura de $35^{\circ} \mathrm{C}$. Não houve efeito do substrato (Tabela 1).

Para porcentagem de germinação, a interação entre os fatores estudados foi significativa. Sementes colocadas para germinar em areia apresentaram maior porcentagem de germinação nas temperaturas de $25^{\circ} \mathrm{C}$, $25-35^{\circ} \mathrm{C}$ e ambiente. Já em vermiculita, apenas a temperatura de $35^{\circ} \mathrm{C}$ apresentou baixa porcentagem de germinação quando comparada com as demais, como se observa na Tabela 1.

Tabela 1 - Porcentagem de germinação e Índice de Velocidade de Germinação (IVG) de diásporos de D. decary, submetidas a diferentes condições de temperaturas e substratos.

\begin{tabular}{|c|c|c|}
\hline Temperatura & IVG & $\begin{array}{l}\text { Porcentagem } \\
\text { de germinação }\end{array}$ \\
\hline 25 & $2,5456 \mathrm{~b}$ & - \\
\hline 30 & $2,5490 \mathrm{~b}$ & - \\
\hline 35 & $0,3751 \mathrm{~d}$ & - \\
\hline $20-30$ & $2,6095 \mathrm{~b}$ & - \\
\hline $25-35$ & $2,1920 \mathrm{c}$ & - \\
\hline Ambiente & $3,0675 \mathrm{a}$ & - \\
\hline \multicolumn{3}{|l|}{ Substratos/temperaturas } \\
\hline Areia $-25^{\circ} \mathrm{C}$ & - & $86,00 \mathrm{a}$ \\
\hline Areia $-30^{\circ} \mathrm{C}$ & - & $75,00 \mathrm{~b}$ \\
\hline Areia -35 & - & $20,80 \mathrm{c}$ \\
\hline Areia $-20-30^{\circ} \mathrm{C}$ & - & $70,80 \mathrm{~b}$ \\
\hline Areia $-25-35^{\circ} \mathrm{C}$ & - & $92,00 \mathrm{a}$ \\
\hline Areia-Ambiente & - & $93,00 \mathrm{a}$ \\
\hline Vermiculita $-25^{\circ} \mathrm{C}$ & - & $76,00 \mathrm{a}$ \\
\hline Vermiculita $-30^{\circ} \mathrm{C}$ & - & $83,00 \mathrm{a}$ \\
\hline Vermiculita $-35^{\circ} \mathrm{C}$ & - & $38,00 \mathrm{~b}$ \\
\hline Vermiculita - $20-30^{\circ} \mathrm{C}$ & - & $87,00 \mathrm{a}$ \\
\hline Vermiculita $-25-35^{\circ} \mathrm{C}$ & - & $80,00 \mathrm{a}$ \\
\hline Vermiculita - Ambiente & - & $91,00 \mathrm{a}$ \\
\hline
\end{tabular}

As médias seguidas da mesma letra não diferem entre si pelo teste de Scott-Knott, a 5\% de probabilidade.
A temperatura de $35^{\circ} \mathrm{C}$, portanto, apresentou resultados inferiores não somente com relação à porcentagem de germinação nos dois substratos testados como também atrasou o período de germinação das sementes. Os resultados evidenciaram, então, que a temperatura elevada e constante de $35^{\circ} \mathrm{C}$ não foi indicada para a germinação das sementes de $D$. decaryi, diferentemente do comportamento de outras palmeiras, como Acoelorraphe wrightii $\mathrm{H}$. Wendl. cujas sementes apresentaram máxima germinação entre 33-39 ${ }^{\circ} \mathrm{C}$ (CARPENTER, 1988); Thrinax morrisii $\mathrm{H}$. Wendl. e Cocothrinax argentata H. Wendl., em temperaturas de 33 e $37^{\circ} \mathrm{C}$ (CARPENTER, 1988; CARPENTER \& GILMAN, 1988), Thrinax parviflora Sw. e Roystonea regia O.F. Cook na própria temperatura constante de $35^{\circ} \mathrm{C}$ (PENARIOL, 2005; PIVETTA et al., 2005a) e, ainda, Syagrus romanzoffiana (Cham) Glassman nas temperaturas de 30 e $35^{\circ} \mathrm{C}$ (PIVETTA et al., 2005b).

As sementes colocadas para germinar em ambiente de laboratório, germinaram mais rápido e apresentaram as mais altas porcentagens de germinação tanto em areia como em vermiculita, numa condição próxima de temperatura alternada de $25-30^{\circ} \mathrm{C}$, que não foi testada neste estudo.

A temperatura de $25^{\circ} \mathrm{C}$, também apresentou maiores porcentagens de germinação das sementes tanto em areia como em vermiculita. Semelhantemente, Aguiar et al. (2005), também obteve maiores porcentagens de germinação de sementes da palmeira Rhapis excelsa Henry ex Rehder em $25^{\circ} \mathrm{C}$ e Iossi et al. (2003) da palmeira Phoenix roebelenii O'Brien em $25^{\circ} \mathrm{Ce} 30^{\circ} \mathrm{C}$.

Ainda neste estudo, para $D$. decaryi, observa-se que a temperatura alternada de $25-35^{\circ} \mathrm{C}$ também apresentou maiores porcentagens de germinação tanto em areia como em vermiculita, semelhante ao encontrado para a germinação de sementes da palmeira Livistona rotundifolia Mart. (VIANA, 2003).

Sementes colocadas para germinar em areia, na temperatura de $35^{\circ} \mathrm{C}$, começaram a germinar por volta do quarto dia após a semeadura e prolongou-se até o trigésimo quinto dia.

O pico de germinação em ambiente e nas temperaturas de $25^{\circ} \mathrm{C}, 30^{\circ} \mathrm{C}$ e $20-30^{\circ} \mathrm{C}$ ocorreram no sexto dia, em ambos os substratos; na temperatura alternada de $25-35^{\circ} \mathrm{C}$ o pico de germinação foi atingido no sexto dia na vermiculita e no oitavo dia na areia; enquanto que na temperatura de $25^{\circ} \mathrm{C}$ o pico de germinação foi no vigésimo sexto dia, para ambos os substratos (Figuras 1 e 2). 


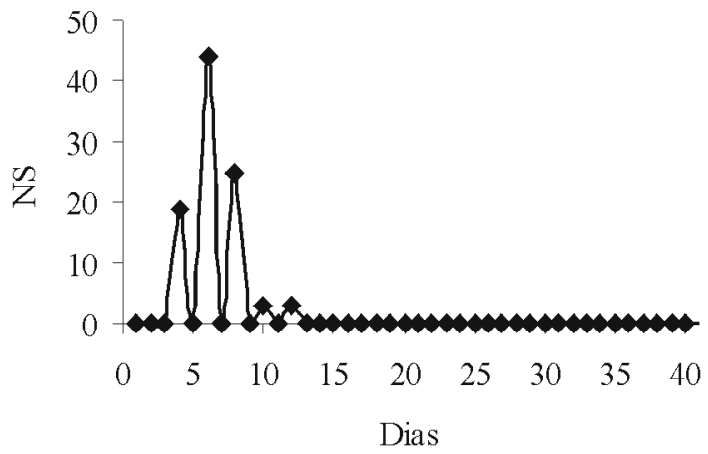

Ambiente

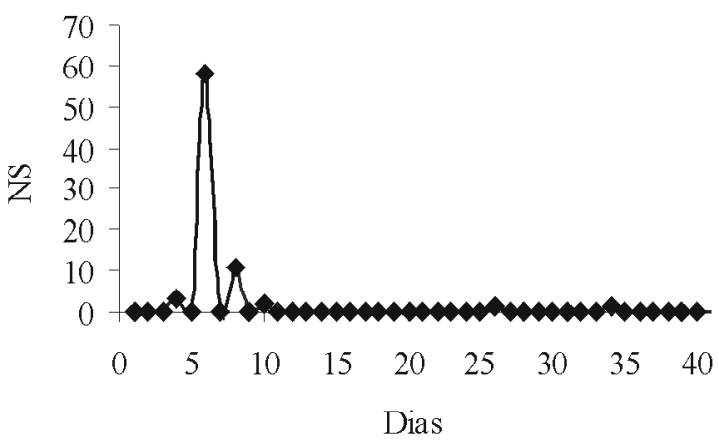

$30^{\circ} \mathrm{C}$

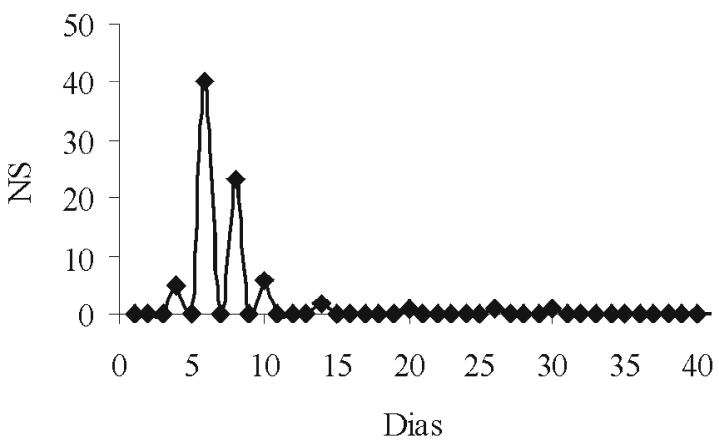

$20-30^{\circ} \mathrm{C}$

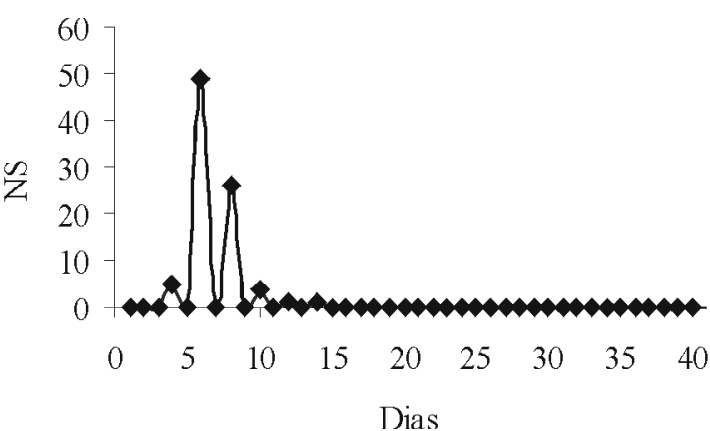

$25^{\circ} \mathrm{C}$

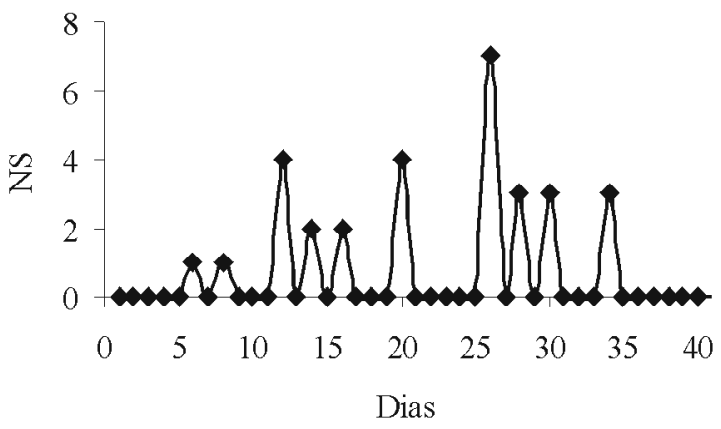

$35^{\circ} \mathrm{C}$

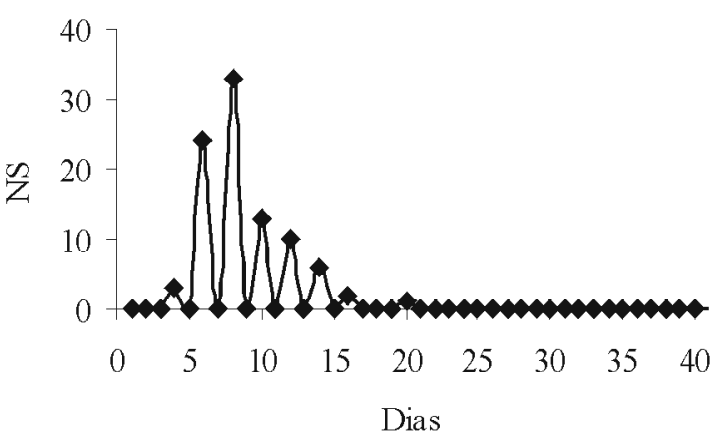

$25-35^{\circ} \mathrm{C}$

Figura 1 - Distribuição da germinação de sementes (NS - número de sementes germinadas/dia) ao longo de 100 dias, de um lote de 100 sementes de Dypsis decary (Jum.) Beentje \& J. Dransf. em diferentes condições de temperatura, tendo como substrato areia. Jaboticabal, SP, 2006.

Sabe-se que a heterogeneidade da germinação dentro de um mesmo lote de sementes distribui o estabelecimento de indivíduos ao longo do tempo e, com isso, a população sobrevive às fases inadequadas ao seu desenvolvimento no campo (CARVALHO \& NAKAGAWA, 2000). Todavia, essa característica não é vantajosa quando se deseja maior homogeneidade de emergência em processos de utilização das sementes em grande escala (NASSIF, 2001). 


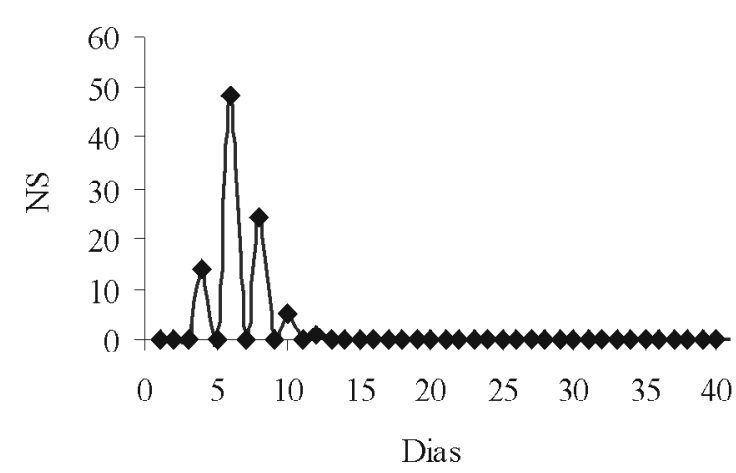

Ambiente

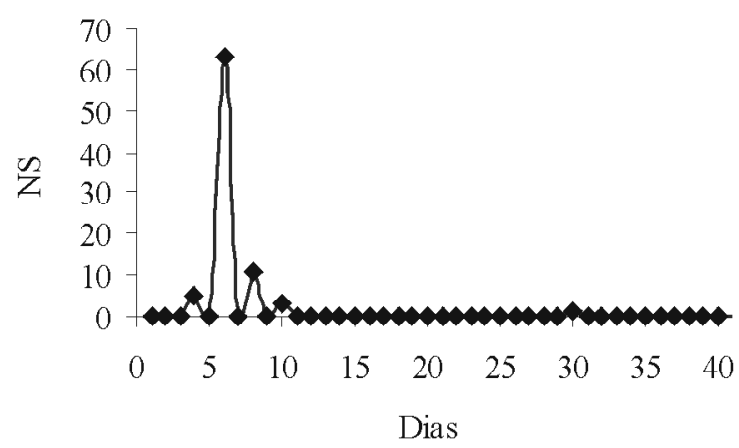

$30^{\circ} \mathrm{C}$

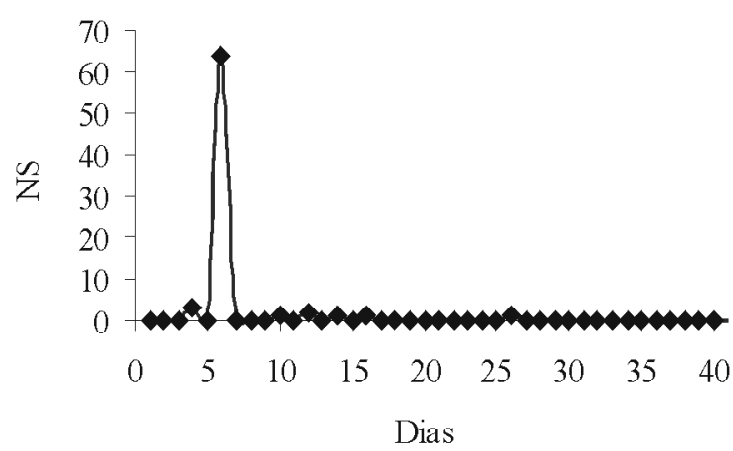

$20-30^{\circ} \mathrm{C}$

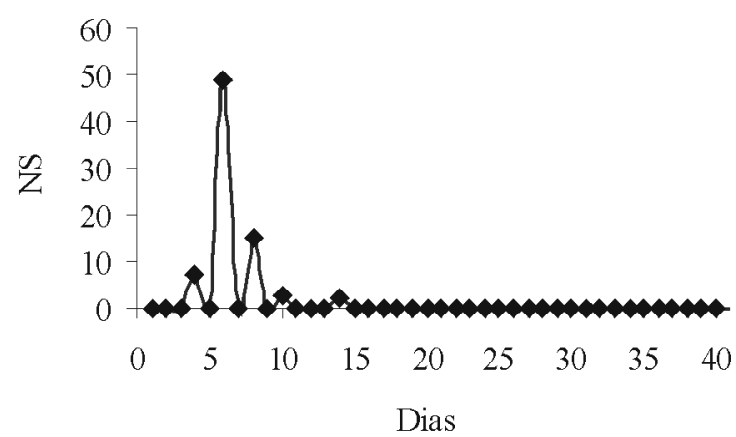

$25^{\circ} \mathrm{C}$

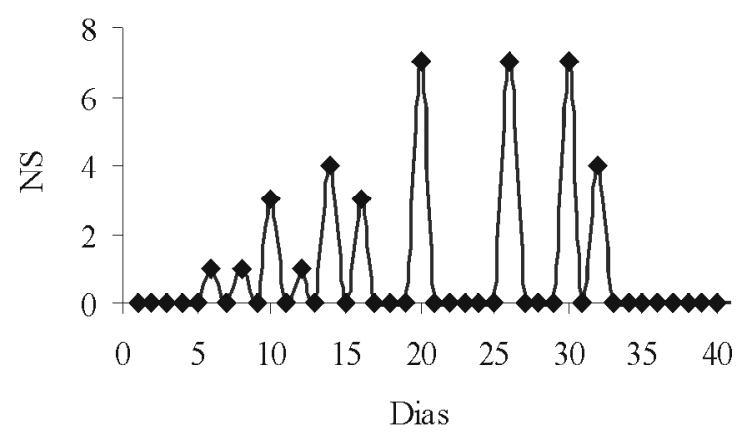

$35^{\circ} \mathrm{C}$

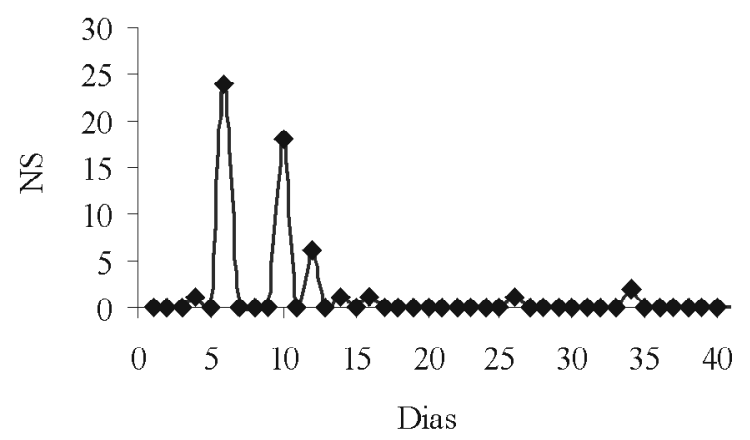

$25-35^{\circ} \mathrm{C}$

Figura 2 - Distribuição da germinação de sementes (NS - número de sementes germinadas/dia) ao longo de 100 dias, de um lote de 100 sementes de Dypsis decary (Jum.) Beentje \& J. Dransf. em diferentes condições de temperatura, tendo como substrato vermiculita. Jaboticabal, SP, 2006

\section{CONCLUSÕES}

De acordo com os resultados, verificou-se que não houve diferença no IVG e na porcentagem de germinação para os substratos testados. A temperatura ambiente foi a que proporcionou o maior IVG das sementes de D. decary. Na temperatura fixa de $35^{\circ} \mathrm{C}$, a porcentagem de germinação e IVG foi bastante reduzida, e o prazo de emergência foi ligeiramente maior, não sendo aconselhada para a germinação dessa espécie. 


\section{AGRADECIMENTOS}

À FAPESP, pelo auxílio-pesquisa.

\section{REFERÊNCIAS BIBLIOGRÁFICAS}

AGUIAR, F. F. A.; BILIA, D. A. C.; KANASHIRO, S.; TAVARES, A. R.; BARBEDO, C. J. Germinação de sementes de Rhapis excelsa (Thunb.) Henry ex Rehder: efeitos da temperatura, luz e substrato. Hoehnea, São Paulo, v. 32, n. 1, p. 119-126, 2005.

BEWLEY, J. D.; BLACK, M. Physiology and biochemistry of seeds. Berlim: Springer-Verlag, 1985. v. 1, 540 p.

BRASIL. Ministério da Agricultura. Regras para análise de sementes. Brasília, DF: Secretaria Nacional de Defesa da Agropecuária, $1992.365 \mathrm{p}$.

BRosChAT, T. K. Palm seed propagation. Acta Horticulturae, Wageningen, v. 360, p. 141-147, 1994.

CARPENTER, W. J. Temperature affects seed germination of four Florida palm species. HotScience, Amsterdam, v. 23, p. 336-337, 1988.

CARPENTER, W. J.; GILMAN, E. F. Effect of temperature and desiccation on the germination of Thrinax morrissi. Proceeding of the Florida State for Horticultural Society, v. 101, p. 288-290, 1988.

CARVALHO, N. M.; NAKAGAWA, J. Sementes: ciência, tecnologia e produção. 4. ed. Jaboticabal: Funep, 2000. 588 p.

COPELAND, L. O.; MCDONALD, M. B. Principle of seed science and technology. New York: Chapmen \& Hall, 1995. 409 p.

FERREIRA, D. F. Análises estatísticas por meio do Sisvar para Windows versão 4.0. In: REUNIÃO ANUAL DA REGIÃO BRASILEIRA DA SOCIEDADE INTERNACIONAL DE BIOMETRIA, 45., 2000, São Carlos. Anais... São Carlos: UFSCar, 2000. p. 255-258.

FIGLIOLIA, M. B.; OLIVEIRA, E. C.; PIÑARODRIGUES, F. C. M. Análise de sementes. In: AGUIAR, I. B.; PIÑARODRIGUES, F. C. M.; FIGLIOLIA, M. B (Coords.). Sementes florestais tropicais. Brasília, DF: ABRATES, 1993. p. 137-174.

IOSSI, E.; SADER, R.; PIVETTA, K. F. L.; BARBOSA, J. C. Efeitos de substratos e temperaturas na germinação de sementes de tamareira-anã (Phenix roebelenii O'Brien). Revista Brasileira de Sementes, Jaboticabal, v. 25, p. 6369, 2003.

LORENZI, H.; SOUZA, H. M.; COSTA, J. T. M.; CERQUEIRA, L. S. C.; FERREIRA, E. Palmeiras brasileiras exóticas e cultivadas. Nova Odessa: Instituto Plantarum, 2004. $416 \mathrm{p}$

MAGUIRE, J. D. Speed of germination aid in selection and evaluation of seedling emergence and vigor. Crop Science, Madison, v. 2, n. 1, p. 176-177, 1962.

MEEROW, A. W. Palm seed germination. Florida: Cooperative Extension Service, 1991. 10 p. (Bulletin, 274).

NASSIF, S. M. L. Aspectos da germinação e emergência de plântulas de Mimosa caesalpiniaefolia Benth. (FABACEAEMIMOSOIDEAE). 2001. 153 f. Tese (Doutorado) Universidade Federal de São Carlos, São Carlos, 2001.

PENARIOL, A. P. Efeito da temperatura e do estádio de maturação na germinação de sementes de Roystonea regia (Kunth) O.F. Cook (Arecaceae). 2005. 32 f. Monografia (Graduação em Agronomia) - Universidade Estadual Paulista, Jaboticabal, 2005.

PIVETTA, K. F. L.; CASALI, L. P.; CINTRA, G. S.; PEDRINHO, D. R.; PIZETTA, P. U. C.; PIMENTA, R. S.; PENARIOL, A. P.; MATTIUZ, C. F. M. Efeito da temperatura e do armazenamento na germinação de sementes de Thrinax parviflora swartz (Arecaceae). Científica, Jaboticabal, v. 33, n. 2, p. 178-184, 2005a.

PIVETTA, K. F. L.; PAULA, R. C.; CINTRA, G. S.; PEDRINHO, D. R.; CASALI, L. P.; PIZETTA, P. U. C.; PIMENTA, R. S. Effects of temperature on seed germination of Queen Palm Syagrus romanzoffiana (Cham.) Glassman. (Arecaceae). Acta Horticulturae, Leuven, v. 683, p. 379-381, 2005 b.

VIANA, F. A. P. Estudos sobre germinação e morfoanatomia do diásporo e da plântula de Livistona rotundifolia (Lam.) Mart. (Arecaceae). 2003. 76 f. Dissertação (Mestrado em Produção e Tecnologia de Sementes) - Universidade Estadual Paulista, Jaboticabal, 2003.

YOCUM, H. G. Factores affecting the germination of palm seeds. American Horticultural Magazine, Washington, v. 43, n. 2, p. 200-201, 1964. 